\title{
Avaliação da Confiança no Funcionamento de Sistemas de Detecção e Alerta de Quedas
}

\author{
Andressa B. Ferreira ${ }^{1,2, *}$, Leonardo S. Piva ${ }^{3}$, Reinaldo B. Braga ${ }^{2,4}$, Rossana M. C. \\ Andrade ${ }^{1,2, * *}$ \\ ${ }^{1}$ Universidade Federal do Ceará (UFC) \\ CEP 60455-900 - Fortaleza - CE - Brasil \\ ${ }^{2}$ Grupo de Redes de Computadores, Engenharia de Software e Sistemas (GREat) \\ CEP 60455-900 - Fortaleza - CE - Brasil \\ ${ }^{3}$ Universidad Miguel Hernandez \\ Alicante - Spain \\ ${ }^{4}$ Instituto Federal de Educação, Ciência e Tecnologia do Ceará (IFCE) \\ CEP 62800-000 - Aracati - CE - Brasil \\ \{andressaferreira, reinaldobraga, rossana\}@great.ufc.br \\ leonardopiva@gmail.com
}

\begin{abstract}
Systems in the area of fall detection and warnings are becoming increasingly popular. However, a high precision on the service provided is necessary, so that the user can accept the system. Therefore, it is important that the system is able to identify normal behavior (i.e., sitting, lying down) and abnormal (i.e., falling), without any loss. Looking to contribute to the achievement of that dependability, this paper presents a set of software quality measurements for the evaluation of the dependability in systems of fall detection. The measurements are collected by an Android system and the results obtained are presented and analyzed.
\end{abstract}

Resumo. Sistemas na área de detecção e alerta de quedas estão se tornando cada vez mais populares. Entretanto, é necessária uma alta precisão no serviço provido pelo sistema para que o usuário possa aceitá-lo e confiar no seu funcionamento. Para tanto, é importante que o sistema seja capaz de identificar comportamentos normais (i.e., sentar, deitar) e anormais (i.e., queda), sem causar qualquer prejuizo ou dano. Buscando contribuir para o alcance dessa confiança, este trabalho apresenta um conjunto de medidas de qualidade de software para a avaliação da confiança no funcionamento em sistemas de detecção e alerta de quedas. As medidas são coletadas em um sistema Android e os resultados obtidos são apresentados e analisados.

\section{Contextualização}

Segundo [Abrantes et al 2013], as quedas em idosos representam um grave problema de saúde pública, devido a fragilidade física de pessoas com mais de 65 anos. Além disso,

\footnotetext{
* Bolsista de mestrado financiada pela Fundação Cearense de Apoio ao Desenvolvimento Científico e Tecnológico

** Bolsista do CNPa de Produtividade DT 2 (número do processo 314021/2009-4)
} 
dados apontam que a população mundial de pessoas com idade superior a 65 anos tem a expectativa de dobrar de 375 milhões em 1990 para 761 milhões em 2025 [Jer-Vui et al 2013]. Com o intuito de auxiliar a vida de pessoas com esse perfil, muitas soluções técnicas têm sido estudadas e desenvolvidas para detectar e alertar quedas.

Os métodos de detecção são normalmente divididos em três tipos [Doughty et al 2000]: dispositivos vestíveis (wearables), dispositivos presentes no ambiente e aplicativos para dispositivos móveis (e.g., smartphones). Apesar da existência de diferenças consideráveis entre os métodos existentes, as soluções têm evoluído ao longo dos anos, buscando diminuir o custo de aquisição do sistema e aumentar a autonomia no processo de detecção e alerta de quedas [Diraco et al 2010].

A autonomia desses sistemas pode ser proporcionada por meio da sensibilidade ao contexto [Doughty et al 2000]. Segundo [Dey e Abowd 1999], um sistema é dito sensível ao contexto quando utiliza informações que caracterizam a situação atual do usuário para prover serviços relevantes.

Com o uso de informações contextuais, o usuário não precisa necessariamente realizar operações diretas (ex.: clicar em um botão para indicar que houve uma queda) para que o sistema forneça o serviço esperado [Santos et al 2013]. Entretanto, delegar o monitoramento de pessoas que necessitam de cuidados especiais a um sistema de software é uma ação que requer confiança no funcionamento (dependability*) do sistema.

Uma das possíveis técnicas para a avaliação da confiança no funcionamento de um sistema é a realização de medições. Uma medição consiste no processo contínuo de definição, coleta e análise de dados sobre o processo de desenvolvimento de software e seus produtos, fornecendo informações significativas com o objetivo de melhorá-los [ISO 25010]. O resultado da medição é chamado de medida [ISO/IEC 9126].

Como apoio à atividade de avaliação da qualidade em sistemas de detecção e alerta de quedas, o objetivo deste trabalho é propor um conjunto de medidas de qualidade de software para a avaliação da Confiança no Funcionamento. Além disso, como prova de conceito, são apresentados os resultados obtidos com a coleta dessas medidas em um sistema Android para detecção e alerta de quedas.

Espera-se, com este trabalho, poder auxiliar a identificação de quais possíveis atributos de qualidade estão impactando a confiança do usuário no funcionamento do sistema e impulsionar melhorias que possibilitem o aumento da aceitação e utilização desse tipo de sistema.

Na seção 2 são apresentadas diversas técnicas encontradas na literatura com o propósito de detectar e alertar quedas, a seção 3 introduz as medidas de qualidade que podem ser utilizadas para a avaliação dos sistemas apresentados, as seções 4 e 5 apresentam um sistema Android para detecção e alerta de quedas e a sua respectiva avaliação com as medidas propostas. Por fim, a seção 6 apresenta a conclusão e os trabalhos futuros.

\footnotetext{
* Neste trabalho, o conceito dependability é traduzido como confiança no funcionamento, baseado em discussões de pesquisadores da área que tentam chegar a um consenso sobre a tradução correta do termo para o português [http://www.cs.kent.ac.uk/people/staff/rdl/CoF/]
} 


\section{Técnicas Existentes para Detecção e Alerta de Quedas}

Como mencionado anteriormente, é possível encontrar diversas soluções para o problema de detecção e alerta de quedas. De acordo com [Doughty et al 2000], a maior diferença entre os vários mecanismos existentes está nos sensores envolvidos no processo de detecção. Segundo [Chen e Chang 2006], quanto menos intrusivo for o conjunto de sensores utilizados, mais promissora é a solução.

As soluções para dispositivo móvel (e.g., [Jer-Vui et al 2013], [Sposaro e Gary et al 2009] e [Piva et al 2014]) são vantajosas, pois são capazes de prover o serviço sem necessidade de custo extra com mudança de hardware ou software. Porém, o funcionamento correto da aplicação está associado ao fato do usuário estar portando o dispositivo junto a si.

Outras técnicas, desenvolvidas para um ambiente específico (e.g., [Yu et al 2012], [Aguilar et al 2014] e [Auvinet et al 2011]), por sua vez, não possuem essa dependência do usuário estar com o dispositivo consigo, porém, o provimento do serviço está restrito a cômodos ou locais determinados, não acompanhando o usuário em todo lugar.

Por fim, apesar de promissores, os sistemas vestíveis (e.g., [Chen et al 2006], [Dumbitrache e Pasca 2013] e [Fudickar et al 2012]) podem ser desconfortáveis dependendo do tamanho ou da posição no corpo. Muitas vezes, dependendo do local onde o dispositivo se encontra alocado e da atividade que o usuário está desempenhando, o sistema pode interpretar movimentos de maneira errônea e gerar alertas incorretos, sendo este um problema também das soluções para dispositivos móveis.

Apesar das diferenças de desenvolvimento e implantação, todas as técnicas tratam os mesmos requisitos não funcionais: confiança e facilidade de uso [Doughty et al 2000]. Para que esses requisitos sejam respeitados, é necessário avaliar o sistema que está sendo proposto e garantir que os requisitos necessários estão sendo respeitados [Ferreira et al 2014]. Essa avaliação pode ser feita com o uso de medidas de qualidade [Ferreira, Braga e Andrade 2014].

A maioria dos trabalhos referenciados aqui propõe as soluções para o problema de detecção e alerta de quedas, deixando a avaliação fora do escopo ou, preocupando-se apenas com a capacidade do sistema de identificar quedas, deixando em segundo plano comportamentos errôneos, por parte do sistema, quando a ação do usuário não foi de fato uma queda.

\section{Medidas de Qualidade de Software Propostas}

As medidas apresentadas neste trabalho foram definidas segundo a metodologia GoalQuestion-Metric (GQM). Essa abordagem é baseada na premissa de que as medições de software devem ser orientadas aos objetivos de medição, que por sua vez devem ser orientados aos objetivos da organização [Basili e Rombach 1994]. Esta técnica é a mais adequada quando o objetivo da medição é claro e específico, como é o caso deste trabalho, onde o objetivo é avaliar a confiança no funcionamento de sistemas de detecção e alerta de quedas. Por esse motivo, essa metodologia foi a escolhida. 
É importante ressaltar também que as medidas apresentadas neste trabalho seguem o formato da norma [ISO/IEC 25000] e são compostas por identificador, nome, definição, função de medição e método de coleta. Essa norma, também conhecida como SQuaRE, é referência quando se trata de medidas de qualidade de software. O seu formato foi adotado nesta pesquisa pois, para que medidas de qualidade sejam consideradas como uma forma de avaliação repetível e aplicável é necessário seguir um formato de padronização.

A Tabela 1 apresenta as medidas propostas. Algumas delas foram retiradas da literatura, pois já se encontravam formatadas de acordo com a [ISO/IEC 25000], outras medidas possuíam apenas nome e descrição, ou nome e função de medição, necessitando, portanto, de uma adaptação. Por fim, algumas medidas foram definidas com base em outras. Informações sobre a origem das medidas e os trabalhos que as embasam podem ser encontradas na última coluna da Tabela 1.

Tabela 1: Medidas

\begin{tabular}{|c|c|c|c|c|c|}
\hline Id & Nome & Descrição & Função de Medição & Interpretação & Ref \\
\hline M1 & $\begin{array}{l}\text { Grau de } \\
\text { Precisão }\end{array}$ & $\begin{array}{l}\text { Representa a capacidade do } \\
\text { sistema de detectar somente } \\
\text { quedas, avaliando, para tal, a } \\
\text { fração de contextos sentidos ou } \\
\text { inferidos que são relevantes para } \\
\text { o sistema. }\end{array}$ & $\frac{P}{P+F P}$ & $\begin{array}{l}\text { Quanto mais } \\
\text { próximo de } 1, \\
\text { melhor. }\end{array}$ & $\begin{array}{l}\text { Adaptada } \\
\text { de [Noury } \\
\text { et al 2007] }\end{array}$ \\
\hline M2 & $\begin{array}{c}\text { Grau de } \\
\text { Sensibilidade } \\
\text { (Recall) }\end{array}$ & $\begin{array}{l}\text { Representa a capacidade do } \\
\text { sistema de detectar quedas, } \\
\text { avaliando, para tal, a fração de } \\
\text { contextos relevantes para o } \\
\text { sistema que foram corretamente } \\
\text { sentidos ou inferidos. }\end{array}$ & $\frac{P}{P+F N}$ & $\begin{array}{l}\text { Quanto mais } \\
\text { próximo de } 1, \\
\text { melhor. }\end{array}$ & $\begin{array}{l}\text { Adaptada } \\
\text { de [Noury } \\
\text { et al 2007] }\end{array}$ \\
\hline M3 & $\begin{array}{c}\text { Grau de } \\
\text { Disponibilidade }\end{array}$ & $\begin{array}{l}\text { Representa a capacidade do } \\
\text { sistema de prestar serviço } \\
\text { quando requisitado. }\end{array}$ & $\frac{T m F}{T m F+T m R}$ & $\begin{array}{l}\text { Quanto mais } \\
\text { próximo de } 1, \\
\text { melhor. }\end{array}$ & $\begin{array}{l}\text { Retirada } \\
\text { de [Lee e } \\
\text { Yun 2012] }\end{array}$ \\
\hline M4 & $\begin{array}{c}\text { Grau de } \\
\text { Especificidade }\end{array}$ & $\begin{array}{l}\text { Representa a fração entre os } \\
\text { alertas não gerados, decorrentes } \\
\text { da ausência de quedas e a soma } \\
\text { destes com o total de alertas } \\
\text { gerados para quedas que não } \\
\text { ocorreram. }\end{array}$ & $\frac{N}{N+F P}$ & $\begin{array}{l}\text { Quanto mais } \\
\text { próximo de } 1, \\
\text { melhor. }\end{array}$ & $\begin{array}{l}\text { Baseada } \\
\text { em M1 e } \\
\text { M2 }\end{array}$ \\
\hline M5 & $\begin{array}{c}\text { Grau de } \\
\text { Desempenho }\end{array}$ & $\begin{array}{l}\text { Representa a média ponderada } \\
\text { entre o Grau de Precisão e o } \\
\text { Grau de Sensibilidade (Recall). }\end{array}$ & $\frac{2 *\left(\frac{P}{P+F P} * \frac{P}{P+F N}\right)}{\frac{P}{P+F P}+\frac{P}{P+F N}}$ & $\begin{array}{l}\text { Quanto mais } \\
\text { próximo de } 1, \\
\text { melhor. }\end{array}$ & $\begin{array}{l}\text { Baseada } \\
\text { em M1 e } \\
\text { M2 }\end{array}$ \\
\hline M6 & $\begin{array}{l}\text { Grau de } \\
\text { Acurácia }\end{array}$ & $\begin{array}{l}\text { Representa a fração de quedas } \\
\text { corretamente sentidos ou } \\
\text { inferidos }\end{array}$ & $\frac{P+N}{F P+P+F N+N}$ & $\begin{array}{l}\text { Quanto mais } \\
\text { próximo de } 1, \\
\text { melhor. }\end{array}$ & $\begin{array}{c}\text { Adaptada } \\
\text { de } \\
\text { [Ranganat } \\
\text { han 2005] }\end{array}$ \\
\hline
\end{tabular}


TmF e TmR representam respectivamente o tempo médio entre falhas e o tempo médio para reparar as falhas. P representa que ocorreu uma queda e o sistema detectou, $\mathrm{N}$ representa que não houve queda e o sistema não detectou, $\mathrm{FP}$ representa que não houve queda e o sistema detectou e, FN representa que houve queda, mas o sistema não identificou.

\section{Prova de Conceito: fAlert}

O fAlert (Fall Alert) é um protótipo de aplicação para smartphones com sistema operacional Android [Piva et al 2014]. O seu funcionamento consiste em utilizar os dados obtidos em tempo real dos sensores acelerômetro, microfone e magnetômetro para detectar uma possível queda.

A Figura 1 mostra o funcionamento do sistema, detalhando as configurações do dispositivo e o processo de ativação do monitoramento dos dados dos sensores.

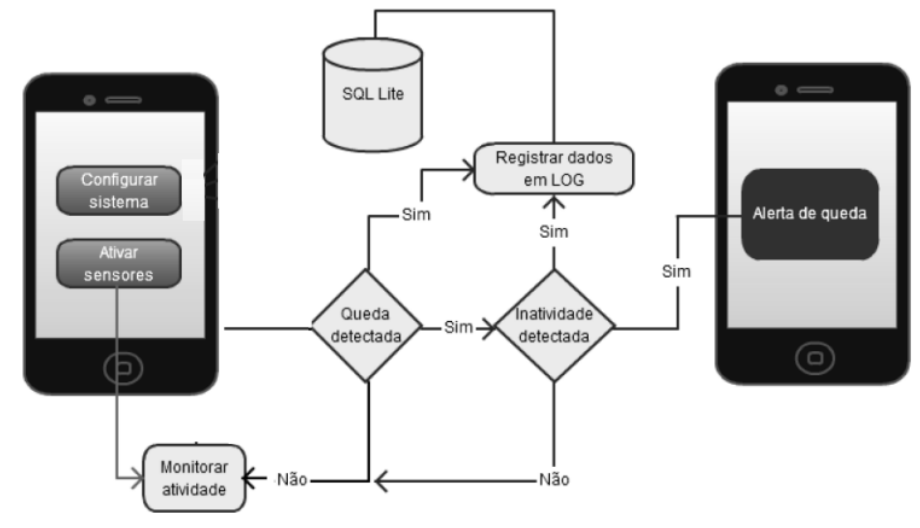

Figura 1 Funcionamento do Sistema fAlert [Piva et al 2014]

Com base na fusão da informação dos três eixos (x, y e z) do acelerômetro e os dados obtidos pela leitura do magnetômetro, a aplicação pode identificar quedas. Isso é possível devido a capacidade do sistema de perceber situações de queda livre seguida da ocorrência de impacto.

Depois de detectada uma possível queda, o sistema ativa o Google Speech Recognizer para identificar se o usuário se encontra bem ou não. A interação deve ocorre em voz alta, por meio do uso de palavras-chave, no tempo máximo de 25 segundos.

No caso do usuário estar bem, a aplicação volta ao seu estado inicial de monitoramento. Caso contrário, quando não há reconhecimento de voz ou o usuário fala que não está bem, o sistema gera o alerta, caracterizado pela realização de uma ligação telefônica para um contato previamente configurado.

\section{Avaliação da Confiança no Funcionamento do Sistema fAlert}

Antes de realizar a avaliação do sistema fAlert foi necessário efetuar diversos testes para que fosse possível reconhecer as atividades rotineiras do usuário, tais como andar, correr, sentar, levantar e deitar. Os testes realizados levaram em consideração diferentes atividades em um ambiente fechado, similar a uma sala de residência. 
Para produzir resultados mais precisos foram realizados 1.140 testes em quatro usuários jovens e saudáveis, utilizando os dispositivos Samsung Galaxy S3 I9300, Samsung Galaxy S3 Slim Duos e $L G$ G2. Estes dispositivos foram colocados em diferentes partes do corpo de cada usuário. No peito, no braço e no bolso, como ilustrado na Figura 2.

Nos testes específicos para os casos de queda foi utilizado um colchão de espuma localizado no chão. Além disso, as ações de cair e deitar foram divididas em categorias: frontal, lateral e para trás, com movimentos lentos, normais ou rápidos.
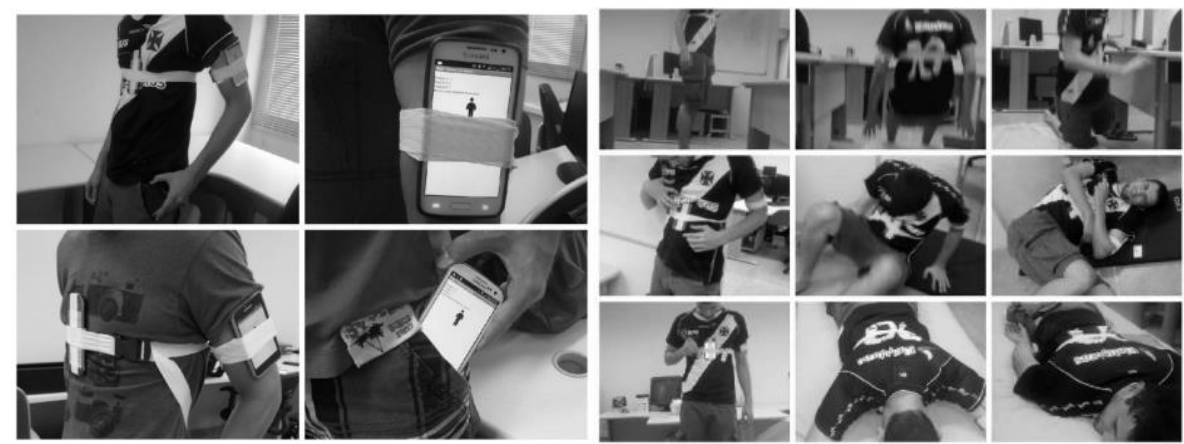

Figura 2: Testes realizados com o fAlert

Os resultados obtidos para a coleta das medidas da Tabela 1, com o dispositivo atado ao peito, estão ilustrados na Figura 3. Os gráficos apresentados representam as medidas coletadas para as três diferentes configurações do sistema, apresentadas na Seção 4: (i) somente acelerômetro; (ii) acelerômetro e microfone; e (iii) acelerômetro, microfone e magnetômetro.
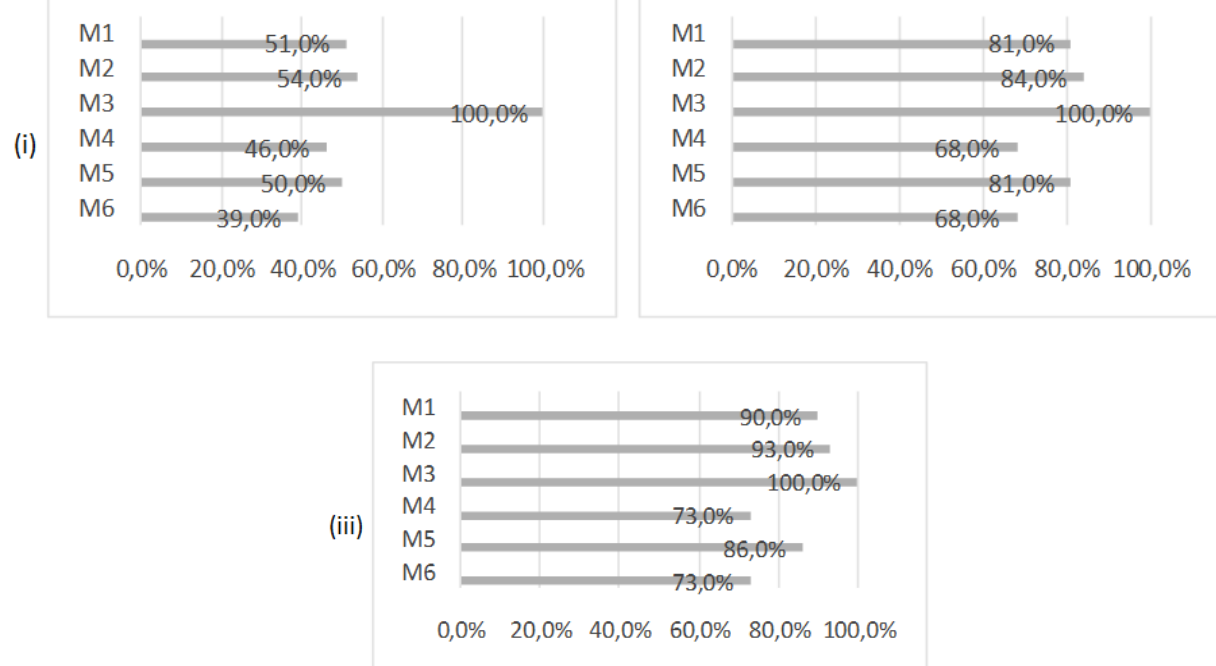

Figura 3: Resultados obtidos com a coleta das medidas para o dispositivo
atado ao peito

Como pode-se observar na Figura 3, quanto mais sensores habilitados durante a configuração do sistema, maiores os valores obtidos com a coleta das medidas. Destacando-se a medida M3 que permanece constante, independentemente da configuração utilizada. Com isso, é possível afirmar que independente dos sensores habilitados, o sistema irá prover o serviço sempre que for necessário. 
Para a medida M1, os valores são sempre inferiores aos das medidas M2 e M3, implicando que a capacidade do sistema de prover o serviço e detectar atividades que podem representar uma queda é superior a sua capacidade de diferenciar as ações do usuário (e.g., diferenciar as ações de deitar rapidamente e cair). A medida M5, por sua vez, é sempre superior as medidas M4 e M6, o que confirma os resultados obtidos com as medidas M1, M2 e M3.

Os demais resultados, para as diferentes posições e movimentos são apresentados na Figura 4 e, buscam minimizar a ocorrência de falsos positivos. Para tanto, é preciso definir qual a melhor posição para a alocação do dispositivo no corpo (peito, braço ou bolso) e qual a melhor configuração (A: acelerômetro, A e Ma: acelerômetro e magnetômetro ou A, Ma e Mi: acelerômetro, magnetômetro e microfone).
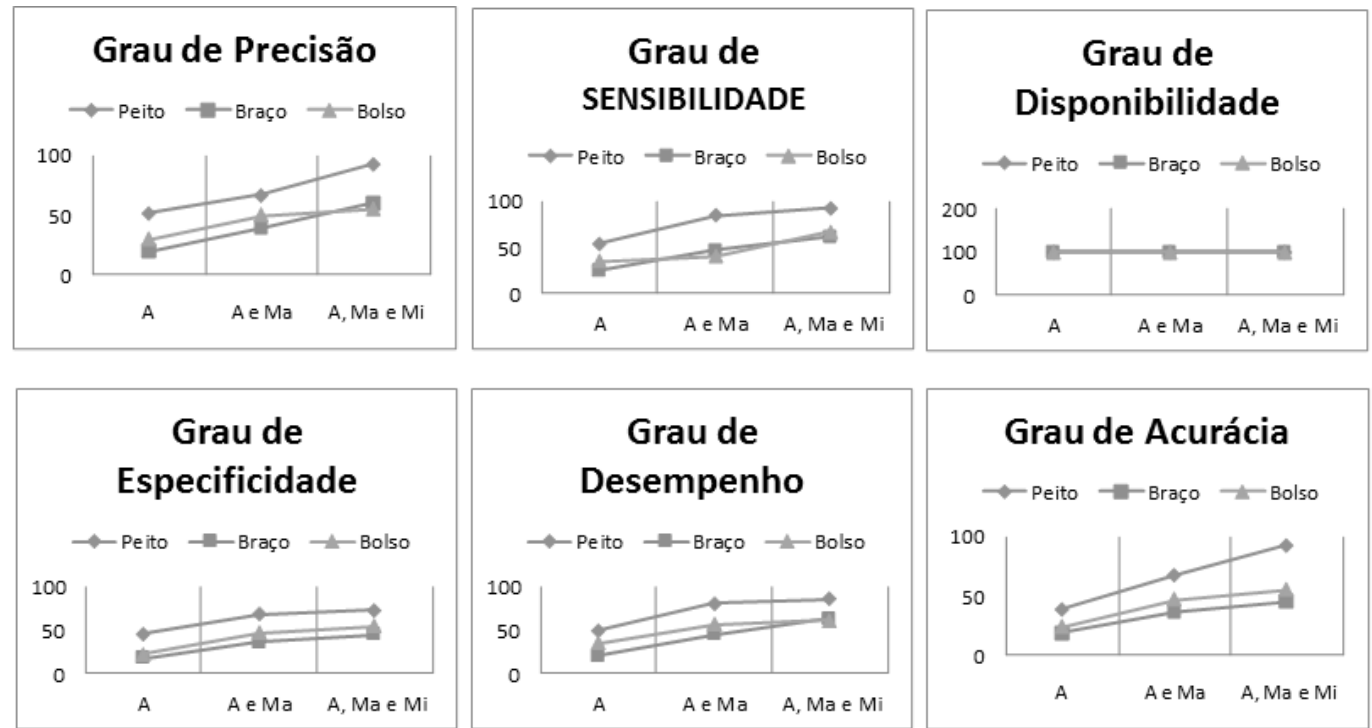

Figura 4: Resultados obtidos para as diferentes posições onde o dispositivo pode ser alocado

Como mencionado na Seção 2, as soluções propostas para dispositivos móveis podem agir de maneira incorreta (e.g., detectar e alertar uma queda com a atividade de deitar) dependendo da localização do dispositivo junto ao corpo do usuário. Além disso, por ser uma solução baseada em threshold, o fAlert não é capaz de reconhecer padrões que identifiquem as atividades cotidianas do usuário, acarretando na possível ocorrência de detecções e alertas para ações que não representam uma queda (e.g., deitar rapidamente).

Como pode-se observar na Figura 4, com a coleta da medida M1, na primeira configuração, é possível perceber, por meio da sua função de medição, que o número de falsos positivos (FP) gerados pelo sistema é significativo. Com isso, ações como sentar, levantar e deitar são incorretamente identificadas como queda. Além disso, independentemente da posição de alocação do dispositivo, mais de $45 \%$ das quedas ocorridas não foram detectadas, reforçando a necessidade do uso da combinação de outros sensores do dispositivo. 
Na segunda configuração, os valores coletados foram melhores, porém, eles não alcançaram os mesmos níveis dos sistemas de detecção de quedas encontrados nos trabalhos de [Sposaro e Gary 2009][Yu et al 2012][Chen e Chang 2012]. Além disso, um número significativo de falsos positivos foi gerado para as ações de sentar, correr e deitar.

Finalmente, na terceira configuração foi possível eliminar, para o celular alocado no peito, as ações de andar, correr, pular, sentar e levantar, pois não houve a geração de falsos positivos. Isso revela indícios de que esta é a melhor posição para alocação de dispositivos com esse tipo de solução.

Com relação a orientação e intensidade das ações do usuário, foi possível perceber, com a coleta das medidas, que atividades laterais ou para trás geram uma quantidade menor de falsos positivos do que ações frontais.

Avaliando a orientação e intensidade das ações realizadas pelo usuário, é possível perceber que situações de queda livre seguidas de impacto são bem identificadas pelo dispositivo alocado no braço, porém, a orientação do dispositivo após o impacto e o ângulo formado entre o celular e o chão influenciam o algoritmo erroneamente.

Quando alocado no bolso, o sistema também gera uma quantidade significativa de falsos positivos. Ao sentar bruscamente o sistema identifica a queda livre e o impacto, após isso, dependendo da altura do usuário, o celular se aloca no bolso de maneira horizontal, caracterizando situações onde a orientação do dispositivo corresponde a uma queda. Além disso, quando a detecção ocorre de maneira correta, a distância da boca até o bolso impede a confirmação sonora, causando atrasos ou erros no processo de detecção.

Quando posicionado junto ao peito, por manter um padrão de movimentos mais estável, independentemente da orientação da atividade ou intensidade relacionada ao movimento, o algoritmo de detecção funciona melhor.

\section{Conclusão e Trabalhos Futuros}

Buscando auxiliar a vida de pessoas que necessitam de cuidados especiais os sistemas de detecção e alerta de quedas vêm se tornando a cada dia mais importantes. Para serem aceitos, é aconselhável que esse tipo de sistema possibilite ao usuário ter um grau satisfatório de confiança no seu funcionamento. É necessário então, levar em consideração vários elementos que podem impactar esta confiança e avaliá-los por meio de medições.

Neste trabalho, medidas de qualidade de software foram propostas e avaliadas por meio da coleta em um sistema Android para detecção e alerta de quedas. A coleta das medidas permitiu a identificação de pontos de melhoria no que tange ao desenvolvimento do sistema fAlert.

Com isso, foi possível proporcionar um aumento significativo no grau de Confiança no Funcionamento do fAlert e, consequentemente, na aceitação do sistema avaliado por parte dos usuários finais. Isso revela indícios de que as medidas propostas são capazes de avaliar características de qualidade, em específico a confiança no funcionamento de um sistema. 
No entanto, entende-se que trabalhos futuros devem ser realizados, são eles: (i) necessidade de novas avaliações, pois as medidas apresentadas neste artigo foram avaliadas tendo como foco apenas um sistema Android para dispositivo móvel, não havendo indícios de que possam ser aplicadas em outros domínios e; (ii) avaliação em sistemas reais, pois o sistema avaliado no estudo de caso é uma prova de conceito (PoC, do inglês proof of concept), logo, não é possível fazer afirmações sobre a aplicação das medidas em sistema reais.

\section{Agradecimentos}

Agradecemos aos membros do GREat Paulo Armando Cavalcante Aguilar, Francisco Eudes Filho, Ricardo Goes de Meira e Gustavo da Silva Monteiro, pelos testes realizados com o sistema fAlert, e ao CAcTUS - "Context-Awareness Testing for Ubiquitous Systems" projeto parcialmente financiado pelo CNPq (Conselho Nacional de Desenvolvimento Científico e Tecnológico), - Universal 14/2013 Número 484380/2013-3, do qual este trabalho é fruto.

\section{Referências}

Abrantes K. S.M. Menezes T. N. Farias2 M. C. A., Silva M. I. L., Rolim V. R. Abreu C. L. "Caracterização das quedas em idosos socorridos pelo serviço de atendimento móvel de urgência”, ABCS Health Science, VOL 38, 2013

Aguilar P. A. C., Boudy J., Istrate D., Dorizzi B., Mota J.C.M., “A Dynamic Evidential Network for Fall Detection", IEEE Journal of Biomedical and Health Informatics, VOL 18, N 4, 2014

Auvinet E., Multon F., Saint-Arnaud A., Rousseau J., and Menunier J., "Fall detection with multiple câmeras: $\mathrm{Na}$ occlusion-resistant method based on 3-d silhouette vertical distribution", IEEE Transactions Information Technology Biomedicine, VOL 15, 2011.

Avizienis, A., Vytautas Magnus Univ., Kaunas, Lithuania, Laprie, J.-C. ; Randell, B., Landwehr, C.; Basic concepts and taxonomy of dependable and secure computing, IEEE Transactions on Dependable and Secure Computing, 2004.

Basili, V., Rombach, H. Goal Question Metric Paradigm. Encyclopedia of Software Engineering - 2, 1994. v. 1p. 528-532

Chen, K. Kwong, Chang D., "Wearable sensors for reliable fall detection", International Conference of the Engineering in Medicine Biology Society, 2006.

Dey, A. K. and Abowd, G. D., "Towards a Better Understanding of Context and Context-Awareness", Proceedings of the 1st international symposium on Handheld and Ubiquitous Computing, 1999.

Diraco, G., Leone A., Siciliano P., "An Active Vision System for Fall Detection and Posture Recongnition in Elderly Healthcare", EDAA, 2010

Doughty K, Lewis R, and McIntosh A M. "The design of a practical and reliable fall detector for community and institutional telecare". Journal of Telemedicine and Telecare, 6(1):150-154, 2000. 
Dumbitrache M., Pasca S., "Fall Detection Algorithm Based on Triaxial Accelerometer Data", IEEE International Conference on E-Health and Bioengineering, 2013

Ferreira, A. B., Piva, L. S., Braga, R. B., Andrade, R. M. C. "Trust Evaluation in an Android System for Detection and Alert Falls". 20th Brazilian Symposium on Multimedia and the Web (WebMedia), 2014.

Ferreira, A. B. ; Braga, R. B. ; Andrade, R. M. C. . Uma Proposta de Medidas de Qualidade para Avaliação da Confiança em Sistemas Ubíquos. In: XII Workshop de Teses e Dissertações em Qualidade de Software, 2014, Blumenau/SC. Anais do XII Workshop de Teses e Dissertações em Qualidade de Software, 2014. p. 31-36.

Fudickar S., Karth C., Mahr P., Schnor B., "Fall-detection Simulator for accelerometers with in-hardware preprocessing", Pervasive Technologies Related to Assistive Environments (PETRA), Greece, 2012

ISO/IEC 25000 (2011). "Software Engineering - Software Product Quality Requirements and Evaluation

ISO/IEC 9126 (2001).“Software Engineering - Product Quality - Part 1”

Jer-Vui L., Yea-Dat C. and Kenny T.H., "Smart Elderly Home Monitoring System with an Android Phone", International Journal of Smart Home Vol. 7, No. 3, 2013.

Lee, J. and Yun, M. H., "Usability Assessment for Ubiquitous Services: Quantification of the Interactivity in Inter-Personal Services. In IEEE International Conference on Management of Innovation \& Technology (ICMIT), 2012.

Miao Y., Adel R., Syed M. N.. "A Posture Recognition-Based Fall Detection System for Monitoring na Elderly Person in a Smart Home Enviroment". IEEE Transactions on Information Technology in Biomedicine, VOL 16, 2012

Noury N., Fleury A., Rumeau P., Bourke A. K., Rialle V., Lundy J. E., "Fall detection Principles and Methods", Proceedings of the 29th Annual International, 2007.

Piva, L. S., Ferreira, A. B., Braga, R. B., Andrade, R. M. C. "fAlert: Um Sistema Android para Monitoramento de Quedas em Pessoas com Cuidados Especiais" Workshop de Ferramentas e Aplicações do XX Simpósio Brasileiro de Sistemas Multimídia e Web, 2014.

Ranganathan, A., Al-Muhtadi, J., Biehl, J., Ziebart, B., Campbell, R. H., Bailey, B., "Towards a Pervasive Computing Benchmark".In Third IEEE International Conference on Pervasive Computing and Communications Workshops, 2005.

Santos M. R., Oliveira K. M., Andrade R. M. C., Santos I. S., Lima E. R.; "A Quality Model for Human-Computer Interaction Evaluation in Ubiquitous Systems" In: 6th Latin American Conference on Human Computer Interaction (CLIHC), 2013.

Scholtz, J. and Consolvo, S., "Toward a Framework for Evaluating Ubiquitous Computing Applications". IEEE Pervasive Computing, 2004.

Sposaro F., Tyson G., "iFall: An Android Application for Fall Monitoring and Response", Florida State University USA, Engineering in Medicine and Biology Society EMBC, Set. 2009. 